\title{
Article
}

\section{The role of answer fluency and perceptual fluency in the monitoring and control of reasoning: Reply to Alter, Oppenheimer, and Epley}

Thompson, Valerie A, Ackerman, Rakefet, Sidi, Yael, Ball, Linden, Pennycook, Gordon and Prowse Turner, Jamie A

Available at http://clok.uclan.ac.uk/9502/

Thompson, Valerie A, Ackerman, Rakefet, Sidi, Yael, Ball, Linden ORCID: 00000002-5099-0124, Pennycook, Gordon and Prowse Turner, Jamie A (2013) The role of answer fluency and perceptual fluency in the monitoring and control of reasoning: Reply to Alter, Oppenheimer, and Epley. Cognition, 128 (2). pp.

256-258. ISSN 0010-0277

It is advisable to refer to the publisher's version if you intend to cite from the work.

http://dx.doi.org/10.1016/j.cognition.2013.03.003

For more information about UCLan's research in this area go to

http://www.uclan.ac.uk/researchgroups/ and search for <name of research Group>.

For information about Research generally at UCLan please go to

http://www.uclan.ac.uk/research/

All outputs in CLoK are protected by Intellectual Property Rights law, including Copyright law. Copyright, IPR and Moral Rights for the works on this site are retained by the individual authors and/or other copyright owners. Terms and conditions for use of this material are defined in the policies page. 


\title{
Running Head: ANSWER AND PERCEPTUAL FLUENCY
}

The role of answer fluency and perceptual fluency in the monitoring and control of reasoning:

Reply to Alter, Oppenheimer, \& Epley (2013)

\author{
Valerie A Thompson* \\ Rakefet Ackerman** \\ Yael Sidi** \\ Linden Ball*** \\ Gordon Pennycook**** \\ Jamie A Prowse Turner*****
}

* University of Saskatchewan, Canada

** Technion-Israel Institute of Technology, Israel

*** University of Central Lancashire, UK

****University of Waterloo, Canada

***** Red Deer College, Canada

Correspondence should be addressed to Valerie Thompson, Department of Psychology, University of Saskatchewan, Saskatoon, SK, Canada, S7N 5A5 or by email: Valerie.Thompson@Usask.Ca 


\begin{abstract}
In this reply, we provide an analysis of Alter, Oppenheimer, and Epley's (2013) response to our earlier paper (Thompson et al., 2013, this issue). In that paper, we reported difficulty in replicating Alter et al.’s (2007) main finding, namely that a sense of disfluency produced by making stimuli difficult to perceive, increased accuracy on a variety of reasoning tasks. Alter et al. (2013) argue that we misunderstood the meaning of accuracy on these tasks, a claim that we reject. We argue and provide evidence that the tasks were not too difficult for our populations (such that no amount of "metacognitive unease" would promote correct responding) and point out that in many cases performance on our tasks was well above chance or on a par with Alter et al.'s (2007) participants. Finally, we reiterate our claim that the distinction between answer fluency (the ease with which an answer comes to mind) and perceptual fluency (the ease with which a problem can be read) is genuine, and argue that Thompson et al. (2013) provided evidence that these are distinct factors that have different downstream effects on cognitive processes.
\end{abstract}


The role of answer fluency and perceptual fluency in the monitoring and control of reasoning: Reply to Alter, Oppenheimer, \& Epley (2013)

Alter, Oppenheimer, Epley, and Eyre (2007) hypothesized that the use of intuitive (Type 1) or analytic (Type 2) reasoning processes could be influenced by a feeling of perceptual disfluency. For example, rendering material difficult to read or asking participants to furrow their brows could create metacognitive unease that would reduce Type 1 and increase Type 2 processing, thereby explaining their finding that accuracy on various reasoning tasks improved under conditions experienced as perceptually disfluent. These intriguing results motivated our follow-up studies, pursued in three independent labs, but with the same goal: to replicate the results and extend them in theoretically relevant ways. We found, however, that we were unable to do this in a straightforward manner. Instead, evidence that perceptual disfluency increased correct responding was elusive, with perceptual disfluency enhancing reasoning accuracy only for those of high cognitive ability (Thompson et al., 2013, this issue). In their comment, Alter et al. (2013) argue that we have misunderstood basic methodological and theoretical concepts, and this explains our discrepant findings. Below we address their main points. What is the meaning of accuracy on reasoning tasks?

Alter et al. (2013) argue that we have mistaken the meaning of accuracy, in particular, by assuming that accuracy (along with confidence) measures deeper processing. We make no such claim but emphasized that accuracy is a poor indicator of Type 2 thinking for many reasons, such as when Type 1 processes produce correct responses (Thompson, 2011; Thompson, Prowse Turner, \& Pennycook, 2011; Thompson et al., 2013, this issue). This is why our study included two different measures of Type 2 thinking: response time and the probability of changing answers. Nowhere did we claim that confidence is a measure of deeper processing. 
What is the effect of perceptual disfluency on metacognitive monitoring?

Alter et al. (2013) claim that, in their 2007 paper, they demonstrated that participants were less confident and more thoughtful about responses when experiencing disfluency. In support of their claim, different groups of participants than those in the main study were asked how confident they were, either while performing a non-reasoning task under disfluent processing conditions, or on the target task, but under instructions not to actually solve the problems. They found that participants anticipated lower confidence in the disfluent than the fluent conditions. In contrast, when we measured reasoners' metacognitive judgments directly, we observed that they were not reduced in disfluent conditions. Specifically, feeling of rightness judgments about intuitive responses were similar in perceptually fluent and disfluent conditions (Experiments 1a and $1 b$ ), as was confidence in final answers (Experiments $3 a$ and $3 b$ ). Thus, the role of perceptual disfluency in metacognitive monitoring remains ambiguous.

What is the effect of perceptual disfluency on analytic engagement?

Thompson et al. (2013, this issue) included two direct measures of analytic engagement: response time and answer changes. Although we did not find increased accuracy, in three out of four experiments where response time was measured, reasoners took longer to think about disfluent stimuli. Importantly, this reflected longer thinking rather than longer reading time. That is, when asked to make quick, intuitive responses (Experiments 1a and 1b), no response time differences arose between fluency conditions. Moreover, the perceptual fluency effect on response times remained after factoring out reading times (Experiments 2a and 2b). However, additional thinking time did not lead to more answer changes or more correct answers, except (in the latter case) for the more cognitively able. As Alter et al. studied undergraduates at elite universities, we proposed that the effect of perceptual disfluency on accuracy depends on 
cognitive ability.

Our results place important boundary conditions on Alter et al.'s (2007) findings:

Researchers searching for more accurate reasoning under disfluent conditions are unlikely to find this with Alter et al.'s tasks, unless testing high ability participants. On the other hand, our data do indicate that perceptual disfluency can trigger analytic thinking when measured by response times. Nonetheless, the perceptual disfluency effect is subtle, given that additional time did not result in answer changes for most participants, nor was it measurable in terms of feeling of rightness or retrospective confidence. We speculated that perceptual fluency may produce a global sense of unease that was strong enough to trigger additional thinking, but which may have prompted most reasoners to justify or rationalise initial answers rather than change them (Stanovich, 2009).

Explaining the discrepancy between Alter et al. (2007) and Thompson et al. (2013, this issue).

Alter et al. (2013) suggest that the items we used were too difficult for our participants, who were consequently unable to solve the tasks regardless of the time they devoted to them. Several of our tasks were modelled closely on Alter et al.'s, such as the CRT (Experiments 2c, 3a and $3 \mathrm{~b}$ ) and the syllogisms task (Experiments 2a and 2b). Alter et al. (2013), however, argue that these tasks may be appropriate for Harvard and Princeton undergraduates, but were beyond the ken of less intellectually elite participants. While we agree that cognitive ability is an important boundary condition on the fluency effect, we disagree that our tasks were too difficult for our participants:

1. In Experiment 3a, we tested a sample from a prestigious university whose average CRT score (2/3 correct) was on par with Alter et al.'s (2007; Experiment 1) sample. These reasoners were clearly up to the challenge, yet we observed a perceptual disfluency effect only 
for those at the higher end of the SAT scale. Thus, contrary to their assertion, high baseline performance levels are insufficient for perceptual disfluency to manifest itself on accuracy.

2. Alter et al. argued that performance on our conditional syllogisms task, used in Experiment 1a, was about at chance levels and well below that observed by Alter et al. (2007) in their Experiment 4. This comparison conflates two quite different tasks: conditional syllogisms (see below) and the quantified syllogisms used by Alter et al. The appropriate comparison is to our Experiments 2a and 2b, where we used quantified syllogisms that were similar to those used by Alter et al. (2007, Experiment 4). Performance on these tasks was well above chance (61\% and $71 \%$ respectively), meaning that the tasks were not, in fact, too difficult for our participants.

3. Finally, we argue that the low accuracy for our conditional syllogisms and remaining CRT experiments does not necessarily imply that these tasks were too difficult for our participants to solve. The conditional syllogism task that we used pits elementary logic (if $\mathrm{p}$, then q; $p$, therefore q?) against belief, and the CRT pits elementary math against fluent pattern recognition. The computational skills and knowledge required to solve these tasks is not beyond the reach of most ordinary college students, e.g. performance on non-misleading versions of the conditional syllogism task are normally above chance (e.g., Schroyens, Schaeken, \& Handley, 2003; Thompson, 1995). The challenge posed by our conditional syllogism task and the CRT is in recognizing that the initial, intuitive, response is incorrect. Our data suggest the experience of perceptual disfluency aids that recognition only for those of high cognitive ability, perhaps because these participants can inhibit the compelling initial response in favour of another. Further research is needed to identify whether this effect is determined by IQ or a correlate of IQ. How many kinds of fluency are there?

We finally consider our evidence that at least two distinct kinds of fluency experiences 
can have different effects on reasoning. One we labelled answer fluency and the other perceptual fluency. Answer fluency involves the production of the answer itself, in particular, how easily it comes to mind. Perceptual fluency concerns the ease with which an entire problem is experienced, as for example, when the font is difficult or participants are encouraged to frown. We argue that the mechanisms producing these two fluency effects are distinct: one arising from the processes used to derive an answer, the other arising from factors external to the problem solving process. On this basis, we predicted that they would have independent and additive effects on metacognitive judgments and reasoning performance.

In contrast, Alter et al. (2013) argued that perceptual fluency is one of the inputs that can influence answer fluency. We disagree fundamentally. The fluency with which answers are produced may be influenced by numerous variables, often labelled as Type 1 processes (e.g., believability, emotionality, availability, habit and relevance), as well as by variables that increase processing speed (e.g., priming, familiarity and expertise). These variables operate regardless of whether the problem is easy or difficult to read. Moreover, in their absence, printing a problem in a fluent font will not bring an answer more quickly to mind. Our data are consistent with this conceptualization. First, when we measured the speed of initial response production (Experiments 1a and 1b) it was the same in the perceptually fluent and disfluent conditions. It was only during the rethinking phase (Experiment $1 b)$ that a difference in response time arose. Second, whereas we observed reliable effects of answer fluency on feeling of rightness judgments, a parallel effect for perceptual fluency was not observed. Moreover, the effects of answer fluency on feeling of rightness and Type 2 thinking were the same, regardless of whether the stimuli were perceptually fluent or not. Collectively, these data are inconsistent with the assertion that perceptual fluency is one of the inputs to answer fluency and instead support our 
hypothesis that they are qualitatively different kinds of fluency effects.

To summarize, building on the Alter et al. findings, we propose a model that advanced the understanding of monitoring and control processes in reasoning and offered an elaborated framework for studying metacognitive processes in reasoning. We believe that one of the fundamental contributions of our paper is in pointing out that fluency effects are diverse, both theoretically and in terms of how they influence reasoning processes. 


\section{References}

Alter, A. L., Oppenheimer, D.M., Epley, N., \& Eyre, R. N. (2007). Overcoming intuition: Metacognitive difficulty activates analytic reasoning. Journal of Experimental Psychology: General, 136, 569-576.

Alter, A.L., Oppenheimer, D., \& Epley, N. (2013). Disfluency prompts analytic thinking-But not always greater accuracy. Cognition [Details provided on publication].

Schroyens, W., Schaeken, W., \& Handley, S. (2003). In search of counter-examples: Deductive rationality in human reasoning. The Quarterly Journal of Experimental Psychology, $56 A(7), 1129-1145$

Stanovich, K. E. (2009). Distinguishing the reflective, algorithmic, and autonomous minds: Is it time for a tri-process theory? In J. Evans \& K. Frankish (Eds.), In Two Minds: Dual Processes and Beyond (pp. 55-88). Oxford: Oxford University Press.

Thompson, V.A. (2005). Conditional reasoning: The necessary and sufficient conditions. Canadian Journal of Experimental Psychology, 49(1), 1-60.

Thompson, V.A. (2011). Normativism vs mechanism. Behavioral \& Brain Sciences, 34, 272273.

Thompson, V.A., Prowse-Turner, J., \& Pennycook, G. (2011). Intuition, Metacognition, and Reason. Cognitive Psychology, 63, 107-140.

Thompson, V.A., Prowse-Turner, J., Pennycook, G., Ball, L.J., Brack, H., Ophir, Y., \& Ackerman, R. (2013). The role of answer fluency and perceptual fluency as metacognitive cues for initiating analytic thinking. Cognition [Details provided on publication]. 